\title{
INSTANTONS FOR DYNAMIC MODELS FROM B TO H
}

\author{
Juha Honkonen $^{\mathrm{a}, *}$, M. V. Komarova ${ }^{\mathrm{b}}$ and M. Yu. Nalimov ${ }^{\mathrm{b}}$ \\ a Theoretical Physics Division, Department of Physical Sciences, \\ P.O. Box 64,FIN-00014 University of Helsinki, Finland \\ b Department of Theoretical Physics, St. Petersburg University, Ulyanovskaya 1, \\ St. Petersburg, Petrodvorets, 198504 Russia
}

\begin{abstract}
Instanton analysis is applied to models $\mathrm{B}-\mathrm{H}$ of critical dynamics. It is shown that the static instanton of the massless $\phi^{4}$ model determines the large-order asymptotes of the perturbation expansion of these near-equilibrium dynamic models leading to factorial growth with the order of perturbation theory.
\end{abstract}

Key words: instanton, dynamic models, large orders PACS: 11.10.Jj, 05.70.Jk

\section{Introduction}

Large-order asymptotic behaviour of perturbation series of the paradigmatic static $\phi^{4}$ field-theoretic model has been thoroughly explored with the aid of instanton analysis and applied to the resummation of the asymptotic series brought about by the standard perturbation expansion $[1,2,3]$.

However, large-order asymptotics in dynamic field theories constructed from Langevin equations with the use of the Martin-Siggia-Rose (MSR) formalism [4] is almost completely unexplored.

\footnotetext{
* corresponding author

Email addresses: Juha.Honkonen@helsinki.fi (Juha Honkonen), komarova@paloma.spbu.ru (M. V. Komarova),

Mikhail.Nalimov@pobox.spbu.ru (M. Yu. Nalimov).
} 
In this paper we use the recently proposed dynamic-instanton method [5] to assess large-order asymptotic behaviour of dynamic models from $\mathrm{B}$ to $\mathrm{H}$ in the standard classification [6]. All these models possess Gibbsian static limit and thus describe dynamics near thermodynamic equilibrium. In the majority of these models mode-coupling effects are present requiring significant development of the method used for model A in Ref. [5]

We construct the stationarity equations for the steepest-descent method and give arguments in favor of existence of the dynamic instanton solution. Largeorder asymptotes of correlation functions, response functions and critical exponents are shown to be determined by the instanton solution of the corresponding static problem, which in all cases considered may be constructed with the aid of the known static instanton of the massless $\phi^{4}$ model.

The present article is organized as follows: in Sec. 2 representation of the MSR field theory in the functional-integral form for the generic model of critical dynamics from the corresponding Langevin equation system is reviewed. Then in Sec. 3 we show that dynamic instanton may be constructed and the steepestdescent evaluation of the functional integral carried out in the same fashion as for model A [5] with the result that the leading contribution is given by the corresponding static instanton. Construction of the dynamic instanton with the aid of the static instanton of the massless $\phi^{4}$ model for models B - H of critical dynamics is analyzed in Sec. 4. Results of this paper are summarized in Sec. 5. Calculation of the fluctuation determinant is reviewed in the Appendix.

\section{Field theory for critical dynamics}

Standard models of critical dynamics may be described by the generic Langevin equation

$$
\frac{\partial \varphi_{a}}{\partial t}+\left(\alpha_{a b}+\beta_{a b}\right) \frac{\delta S}{\delta \varphi_{b}}=\xi_{a}
$$

determining dynamics of fluctuations in a system with the thermodynamic equilibrium corresponding to the field theory with the static "action" (effective Hamiltonian) $S$. In (1) the source $\xi$ is a Gaussian random field with zero mean and the correlation function

$$
\left\langle\xi_{a}(t, \mathbf{x}) \xi_{b}\left(t^{\prime}, \mathbf{x}^{\prime}\right)\right\rangle=2 \alpha_{a b} \delta\left(t-t^{\prime}\right) \delta\left(\mathbf{x}-\mathbf{x}^{\prime}\right)
$$

chosen to maintain the fluctuation-dissipation theorem. To this end the following conditions are imposed on the terms of the kinetic coefficient as well:

$$
\alpha^{\top}=\alpha, \quad \beta^{\top}=-\beta, \quad \frac{\delta \beta_{a b}}{\delta \varphi_{a}}=0
$$


The symmetric part of the kinetic coefficient $\alpha$ is assumed to be field-independent and positive-definite. In equation (1) the static action is assumed to have threepoint and four-point interaction terms only and to contain the action of the massless $\phi^{4}$ model

$$
S_{4}=\frac{1}{2}(\nabla \phi)^{2}+\frac{g}{4 !} \phi^{4},
$$

for which the instanton solution is known [1]. Relations (1) - (3) constitute a compact form of the description of all standard models of critical dynamics [7]. Here and henceforth, all necessary integrals and sums are implied. In the forthcoming treatment the full $d$-dimensional Euclidian space $\mathbf{R}^{d}$ is assumed for spatial variables, but for the time variable it is convenient to carry out most of the analysis on a finite time interval $\left[t_{0}, T\right]$ and pass to the limit $t_{0} \rightarrow-\infty$ and $T \rightarrow \infty$ at a later stage.

In the MSR approach, functional integrals for correlation and response functions are calculated with the "measure"

$$
\mathfrak{M}\left[\varphi, \varphi^{\prime}\right]=\mathcal{D} \varphi \mathcal{D} \varphi^{\prime} \sqrt{\operatorname{det} M_{0}^{T} M_{0}} e^{-\bar{S}}
$$

where the dynamic action for the model (1) - (3) may be written symbolically [4] as

$$
\bar{S}=-\varphi_{a}^{\prime} \alpha_{a b} \varphi_{b}^{\prime}+\varphi_{a}^{\prime}\left[\frac{\partial \varphi_{a}}{\partial t}+\left(\alpha_{a b}+\beta_{a b}\right) \frac{\delta S}{\delta \varphi_{b}}\right] .
$$

We stick to the choice of Ref. [5] of the determinant factor in representation (5) in the field-independent form with the self-adjoint operator $M_{0}^{T} M_{0}$, where the operator $M_{0}$ is generated by the free-field part of dynamic action (6) as

$$
\left(M_{0}\right)_{a b}=\delta_{a b} \frac{\partial}{\partial t}+\left(\alpha_{a c}+\beta_{a c}^{(0)}\right) K_{c b}
$$

Here, $\beta^{(0)}$ is the field-independent part of the skew-symmetric term of the kinetic coefficient, whereas $K_{a b}$ is the kernel of the quadratic form $S_{0}$ of the static action:

$$
S_{0}=\frac{1}{2} \varphi_{a} K_{a b} \varphi_{b}
$$

and is thus, by definition, symmetric and positive. The choice of the determinant factor in measure (5) implies that the interaction functional $\bar{S}_{I}$ is taken in the normal form, which in the perturbation theory is equivalent to the condition that there are no graphs with closed loops of the retarded propagators $\left(M_{0}^{-1}\right)_{a b}\left(t, \mathbf{x} ; t^{\prime}, \mathbf{x}^{\prime}\right)$ attached to the interaction vertex. This amounts to vanishing retarded free-field propagator at coinciding times, and no fielddependent factors to the determinant $\operatorname{det} M_{0}^{T} M_{0}$ are generated.

We remind that initially in the ordinary perturbation theory the boundary conditions on the time axis fix $\varphi^{\prime}(T)=0$, but leave $\varphi(T), \varphi^{\prime}\left(t_{0}\right)$ and $\varphi^{\prime}(T)$ arbitrary. In the instanton approach the space of integration $E(\Delta)$ in the 
functional integral is chosen using the properties of the quadratic form of the expansion of dynamic action at the saddle point and therefore it is in general different from that of the ordinary perturbation theory, the construction being similar to that for model A [5].

\section{Instanton analysis for the generic model of critical dynamics}

We are dealing with a dynamic model which has several coupling constants which, however, are proportional to some powers of a single parameter, e.g. $\epsilon$, at the fixed point of the renormalization group equation). Therefore, it seems most reasonable to base the large-order analysis on the number of loops rather than powers of different coupling constants. This may be effected by extracting a common factor $u$ from all coupling constants and then counting large orders by powers of $u$. Thus, we cast the interaction part of the static action in the form

$$
v_{a b c} \varphi_{a} \varphi_{b} \varphi_{c}+g_{a b c d} \varphi_{a} \varphi_{b} \varphi_{c} \varphi_{d}=u \bar{v}_{a b c} \varphi_{a} \varphi_{b} \varphi_{c}+u^{2} \bar{g}_{a b c d} \varphi_{a} \varphi_{b} \varphi_{c} \varphi_{d}
$$

The task then amounts to a steepest-descent calculation of the parametric integral

$$
\frac{1}{2 \pi i} \oint \frac{d u}{u} \frac{\iint \mathcal{D} \varphi \mathcal{D} \varphi^{\prime} \Phi_{a_{1}}\left(t_{1}, \mathbf{x}_{\mathbf{1}}\right) \ldots \Phi_{a_{k}}\left(t_{k}, \mathbf{x}_{\mathbf{k}}\right) e^{-\bar{S}-N \ln u}}{\iint \mathcal{D} \varphi \mathcal{D} \varphi^{\prime} e^{-\bar{S}_{0}}}
$$

where $\Phi=\left\{\varphi, \varphi^{\prime}\right\}$, expressing the $N$ th order contribution to perturbation expansion in $u$ of the $k$-point Green function. In representation (10) the normalizing determinant has been written in the form of a functional integral with the free-field part of the dynamic action in order to make explicit cancellation of Jacobians related to various changes of variables. As shown in Ref. [5], an instanton solution for expression (10) for model A may be constructed in close relation to the instanton solution of the corresponding equilibrium model. In this Section we show that a similar procedure allows to reduce the large-order analysis of the dynamic functional integral (10) to that of the static problem determined by the static action $S$.

It is convenient to extract the large factor $N$ in the exponential of the numerator of expression (10) by a suitable change of variables accompanied by the same transformation in the denominator to avoid uninteresting Jacobi deter-

minants: $\varphi_{a} \rightarrow \sqrt{N} \varphi_{a}, \varphi_{a}^{\prime} \rightarrow \sqrt{N} \varphi_{a}^{\prime}, u \rightarrow u / \sqrt{N}$. To simplify the forthcoming calculation of the fluctuation integral around the saddle point we also scale out the dependence on the variable $u$ by $\varphi_{a} \rightarrow \varphi_{a} /(i u), \varphi_{a}^{\prime} \rightarrow \varphi_{a}^{\prime} /(i u)$. As a result of these transformations we arrive - up to the factor $N^{(N+k) / 2}(i u)^{-k}$ 
- at the representation

$$
\frac{1}{2 \pi i} \oint \frac{d u}{u} \frac{\iint \mathcal{D} \varphi \mathcal{D} \varphi^{\prime} \Phi_{a_{1}}\left(t_{1}, \mathbf{x}_{\mathbf{1}}\right) \ldots \Phi_{a_{k}}\left(t_{k}, \mathbf{x}_{\mathbf{k}}\right) e^{-N\left(-\bar{S} / u^{2}+\ln u\right)}}{\iint \mathcal{D} \varphi \mathcal{D} \varphi^{\prime} e^{N \bar{S}_{0} / u^{2}}}
$$

where the dynamic action $\bar{S}$ (as well as the static action $S$ ) are independent of the loop-counting variable $u$ and these can be obtained from the initial ones by change of the coupling constants $g_{a b c d} \rightarrow-\bar{g}_{a b c d}, v_{a b c} \rightarrow-i \bar{v}_{a b c}$.

The stationarity equations of the method of steepest descent assume the form

$$
\begin{aligned}
\frac{\delta \bar{S}}{\delta \varphi_{a}} & =-\frac{\partial \varphi_{a}^{\prime}}{\partial t}+\varphi_{b}^{\prime}\left(\alpha_{b c}+\beta_{b c}\right) \frac{\delta^{2} S}{\delta \varphi_{c} \delta \varphi_{a}}+\varphi_{b}^{\prime} \frac{\delta \beta_{b c}}{\delta \varphi_{a}} \frac{\delta S}{\delta \varphi_{c}}=0 \\
\frac{\delta \bar{S}}{\delta \varphi_{a}^{\prime}} & =-2 \alpha_{a b} \varphi_{b}^{\prime}+\frac{\partial \varphi_{a}}{\partial t}+\left(\alpha_{a b}+\beta_{a b}\right) \frac{\delta S}{\delta \varphi_{b}}=0 \\
\bar{S} & =-\frac{u^{2}}{2}
\end{aligned}
$$

As in static theory, equations (12) - (14) have a nontrivial solution with negative $u^{2}$ only. The dynamic instanton $\varphi_{D}$ for the basic field is the nontrivial solution of the equation

$$
-\frac{\partial \varphi_{a}}{\partial t}+\left(\alpha_{a b}-\beta_{a b}\right) \frac{\delta S}{\delta \varphi_{b}}=0 .
$$

Substitution of this solution to equation (13) leads to the nontrivial instanton $\varphi_{D}^{\prime}$ for the auxiliary field, whose equation we quote in the two equivalent forms used later:

$$
\varphi_{D a}^{\prime}=\frac{\delta S}{\delta \varphi_{a}}=\left(\alpha^{-1}\right)_{a b}\left(\frac{\partial \varphi_{b}}{\partial t}+\beta_{b c} \frac{\delta S}{\delta \varphi_{c}}\right) .
$$

With the use of the instanton equation (15) and the antisymmetry property of the kinetic coefficient $\beta_{a b}$ it may be readily seen that the stationarity equation (12) is fulfilled on solution (16).

In addition to equations (12), (13) and (14) variation on the boundaries of the time interval produces [assuming fixed initial field $\left.\varphi\left(t_{0}, \mathbf{x}\right)\right]$ the condition

$$
\varphi^{\prime}(T, \mathbf{x})=0
$$

which - due to the first equation (16) - singles out the solution approaching the static instanton at the final time instant. Indeed, according to relations (16) and (17) the dynamic instanton $\varphi_{D}$ is a function conforming to the Cauchy condition for the final time instant $\varphi_{D}(T, \mathbf{x})=\varphi_{s t}(\mathbf{x})$, where $\varphi_{s t}$ is the static instanton solution for the static action $S$. It should be noted that the iterative solution of the instanton equation (15) also has the property $\lim _{t \rightarrow-\infty} \varphi_{D}(t, \mathbf{x})=$ 
0 , because - due to the sign changes in comparison with (13) — the Green's function of linear part of equation (15) vanishes infinitely far in the past. A similar equation for the model $\mathrm{A}$ was investigated in [5], where it was shown that the solution of this equation on the infinite time range for negative $u^{2}$ tends to zero at $t \rightarrow-\infty$ and to $\varphi_{s t}$ at $t \rightarrow \infty$.

Substitution of the solution $\varphi_{D}$ and $\varphi_{D}^{\prime}$ in dynamic action (6) reveals that the dynamic action on the dynamic instanton solution gives rise to a time integral of a total time derivative (the expression $\partial S / \partial t=(\partial \varphi / \partial t)(\delta S / \delta \varphi)$ was used) asymptotically coincides with the static action on the static instanton:

$$
\bar{S}\left(\varphi_{D}, \varphi_{D}^{\prime}\right)=S\left(\varphi_{s t}\right)-S\left(\varphi_{0}\right) \underset{t_{0} \rightarrow-\infty}{\longrightarrow} S\left(\varphi_{s t}\right)
$$

here $\varphi_{0}(\mathbf{x})=\varphi_{D}\left(t_{0}, \mathbf{x}\right)$. Equation (15) gives rise to iterative solution with an advanced Green's function, therefore the field $\varphi_{0}$ vanishes in the limit $t_{0} \rightarrow-\infty$

Similar the third saddle-point equation (14) on solution (15) and (16) reduces to the following equation for the limiting values of the dynamic instanton

$$
S\left(\varphi_{s t}\right)-S\left(\varphi_{0}\right)=-\frac{u^{2}}{2}
$$

and thus recovers the stationarity equation for the static instanton

$$
S\left(\varphi_{s t}\right)=-\frac{u^{2}}{2}, \quad t_{0} \rightarrow-\infty
$$

Thus, as for model A, we arrive at the conclusion that the exponential factor in our steepest descent analysis of Green function (11) as well as pre-exponential factor asymptotically are the same as in the corresponding equilibrium static theory, provided there is a nontrivial solution of equation (15). Such a solution may be constructed in the same fashion as in model A as the usual tree-graph solution of the nonlinear equation (15) with the given final Cauchy condition $\varphi_{D}(T, \mathbf{x})=\varphi_{s t}(\mathbf{x})$ instead of an initial condition.

In the integration space of the functional integral of the $k$-point Green functions (11) the degeneracies of the instanton due to the usual dilatation invariance and translation invariance in coordinate space may be removed with the aid of the same unit decomposition $[1,10,11]$ as in the static instanton theory with the corresponding conditions imposed on on the final value of the integration field $\varphi(T, \mathbf{x})$. 
Thus, we arrive at the Green function

$$
\frac{1}{2 \pi i} \oint \frac{d u}{u} \frac{\iint \mathcal{D} \varphi \mathcal{D} \varphi^{\prime} \Phi_{a_{1}}\left(t_{1}, \mathbf{x}_{\mathbf{1}}\right) \ldots \Phi_{a_{k}}\left(t_{k}, \mathbf{x}_{\mathbf{k}}\right) \mathrm{I} e^{-N\left(-\bar{S} / u^{2}+\ln u\right)}}{\iint \mathcal{D} \varphi \mathcal{D} \varphi^{\prime} e^{N \bar{S}_{0} / u^{2}}}
$$

where I stands for all contributions from the degeneracy-lifting unit decomposition. At the leading order in $N$ we replace all $\Phi\left(t_{i}, \mathbf{x}_{\mathbf{i}}\right)$ by $\left\{\varphi_{D}, \varphi_{D}^{\prime}\right\}$ in the pre-exponential factor. In the stationary limit $t_{0} \rightarrow-\infty$ the initial value of the instanton $\varphi_{D}\left(t_{0}\right)$ vanishes and in the exponential the static instanton action is recovered at leading order in $N$.

The most subtle point of the saddle-point approach, i.e. the calculation of the fluctuation determinant at the instanton solution, is outlined in the Appendix. The main conclusion is - as in the case of the model A [5] - that the fluctuation determinant coincides with that of the static theory. The analysis of large-order contributions to correlation and response functions also proceeds in the same way as for model $\mathrm{A}$ and we do not dwell on it here.

Thus, we conclude that in the translation-invariant in time case the asymptotic properties of the dynamic model at leading order in $N$ are determined by the static instanton solution which leads to factorial growth of the large-order contributions as in the static instanton analysis. Therefore, the large-order behaviour of an arbitrary quantity $F$ (correlation or response function or critical index) may be expressed as

$$
F^{[N]}=C N ! a_{M}^{N} N^{b}
$$

where $F^{[N]}$ is the $N$ th order contribution to $F$ of the expansion in the parameter $e$ ( $e$ is the coupling constant $g$ or the dimensional regularization parameter $\epsilon)$. In (20) the constant $a_{M}$ depends on the model only $(M=A, B, C, \ldots)$, whereas the exponent $b$ and the amplitude factor $C$ - either a constant or a function of coordinate and time arguments - depend on the quantity $F$ as well. See, for instance, the analysis of the factor $C$ for a generic function in the static theory presented in Ref. [12]).

As in the case of the model A [5], when expression (20) is used for contributions to correlation or response functions, the $\Phi$ fields in the pre-exponential factor of (19) have to be replaced by the static instanton $\varphi_{s t}$ for the fields $\varphi$ and put equal to zero for the fields $\varphi^{\prime}$ at the leading order in $1 / N$. The dynamic properties of the Green functions are thus determined by the first correction in $1 / N[5]$. 


\section{Static and dynamic instantons for models $\mathrm{B}-\mathbf{H}$}

We have shown in Sec. 3 that at the leading order the results of the dynamicinstanton calculation are determined by the static instanton corresponding to the static action $S$. In this Section we review the results of the static-instanton analysis, which in all cases are based on the long-known static instanton of the massless $\phi^{4}$ model.

\subsection{Model B}

In model B only one field is present, the static action is that of the massless $\phi^{4}$ model (4) and the symmetric kinetic coefficient is proportional to the Laplace operator:

$$
\alpha=-\lambda \nabla^{2}
$$

Since the static action is exactly the same as in model A and the large-order behaviour of the perturbation expansion is determined by the static instanton, the conclusions about this behaviour are the same as for model A: in model B expression (20) is valid with the constant $a_{B}=a_{0}$ known from the analysis of the static theory $[1,10]$ [for the action $\left.(4) a_{0}=1 /\left(16 \pi^{2}\right)\right]$.

Although the explicit form of the dynamic instanton was not used in the analysis presented above it would be useful for the investigation of the amplitudes $C$ in relation (20) for the dynamic Green functions. Therefore, we remind how the dynamic instanton may be constructed. A convergent iterative solution of the equation (15) for model $\mathrm{B}$ can be written in analogy with model A [5], viz.

$$
\phi_{D}^{(0)}(t, \mathbf{x})=\int d \mathbf{x}^{\prime} \widetilde{G}\left(T-t, \mathbf{x}-\mathbf{x}^{\prime}\right) \phi_{s t}\left(\mathbf{x}^{\prime}\right)
$$

can be taken as the zeroth-order approximation. The next orders of the expansion are constructed as the usual tree-graph solution of equation (15) with the aid of the advanced kernel of the linear operator $\left(\partial_{t}-\lambda \nabla^{4}\right)$, which can be represented in the form

$$
\widetilde{G}(t, \mathbf{x})=-\Theta(-t) \int_{-\infty}^{\infty} d y \frac{1}{(2 \pi)^{d} \sqrt{4 \pi \lambda t}}\left(\frac{\pi}{i y}\right)^{d / 2} e^{-\frac{y^{2}}{4 \lambda t}+\frac{i \mathbf{x}^{2}}{4 y}}
$$




\subsection{Model C}

In model C there are two components of the basic field: let $\varphi_{1}=\phi$ and $\varphi_{2}=m$, and the static action is of the form:

$$
S_{C}=\frac{1}{2}(\nabla \phi)^{2}+\frac{g}{4 !} \phi^{4}+\frac{m^{2}}{2}+\frac{1}{2} v_{2} m \phi^{2} .
$$

The kinetic coefficient is still symmetric and diagonal

$$
\alpha=\left(\begin{array}{cc}
\Gamma & 0 \\
0 & -\lambda \nabla^{2}
\end{array}\right) .
$$

To keep track of the number of loops we put $g=u^{2}$ and $v_{2}=\varkappa u$. Then the static instanton is determined by the set of equations

$$
\begin{aligned}
-\nabla^{2} \phi-\frac{1}{6} \phi^{3}-i \varkappa m \phi & =0, \\
m-\frac{i}{2} \varkappa \phi^{2} & =0, \\
S_{C} \mid \begin{array}{c}
g=-1 \\
v_{2}=-i \varkappa
\end{array}=-\frac{u^{2}}{2}, &
\end{aligned}
$$

and thus may be constructed with the use of the usual instanton solution $\phi_{s t}$ of the massless $\phi^{4}$ model:

$$
\begin{aligned}
-\nabla^{2} \phi_{s t}-\frac{1}{6} \phi_{s t}^{3} & =0, \\
\frac{1}{2} \int d \mathbf{x}\left(\nabla \phi_{s t}\right)^{2} \phi_{s t}-\frac{\tilde{g}}{4 !} \int d \mathbf{x} \phi_{s t}^{4} & =-\frac{u^{2}}{2},
\end{aligned}
$$

with $\tilde{g}=3 \varkappa^{2}-1$. To use large-order asymptotes for investigation of the $\epsilon$ expansion we write $\varkappa=\varkappa(\epsilon)=v_{2 *} / \sqrt{g_{*}}$ ( where $v_{2 *}$ and $g_{*}$ are the coordinates of a fixed point of the renormalization-group flow equations).

The stability of the fixed point in the model considered depends on the value of the critical index of the specific heat $\alpha$. If the index $\alpha<0$, then $v_{2 *}=0$ at the stable fixed point. In this case $\tilde{g}=-1$ and the constant $a=a_{C}$ in expression (20) for this model is the same as in the model A and the usual static $\phi^{4}$ model [1,2]: $a_{C}=a_{0}$.

In the opposite case, when index $\alpha>0$ we have $g_{*} \sim \epsilon$ and $v_{2 *}^{2} \sim \epsilon$ at the stable fixed point giving rise to a nontrivial $\epsilon$ expansion for the parameter $\varkappa=v_{2} / u[7]$ :

$$
\varkappa=\varkappa^{(0)}+\varkappa^{(1)} \epsilon+\varkappa^{(2)} \epsilon^{2}+\ldots
$$


Therefore, the constant $a_{C}$ in relation (20) is now determined by the equation

$$
\frac{1}{a_{0}}=\frac{\left[1-3 \varkappa(\epsilon)^{2}\right]}{a_{C}}
$$

Taking into account that in the $\epsilon$ expansion asymptotic structure (20) appears as

$$
F^{[N]} \epsilon^{N}=N ! C a_{C}^{N} N^{b} \epsilon^{N}
$$

we see that the only contribution to the constant $a_{C}$ comes from the $\varkappa^{(0)}$ term of expansion (30). In this case, therefore,

$$
a_{C}=\frac{a_{0}}{1-3 \varkappa^{(0) 2}} .
$$

The coefficient $\varkappa^{(1)}$ from expression (30) determines the correction to the amplitude factor $C$ of the form

$$
\exp \left[\frac{6 \varkappa^{(0)} \varkappa^{(1)}}{1-3 \varkappa^{(0) 2}}\right]
$$

whereas $\varkappa^{(2)}$ and higher-order terms of the $\epsilon$ expansion of $\varkappa(\epsilon)$ are irrelevant for the leading order in $1 / N$.

Let us note that actually we are considering here a $\sqrt{\epsilon}$ expansion, since the loop-counting parameter $u \sim \sqrt{\epsilon}$ at the leading order, and due to the scaling $\phi, m \rightarrow \phi /(i u), m /(i u)$ the $u$ expansion of correlation functions with an odd number of $m$ fields contains odd powers of $u$ whereas those with an even number of $m$ fields contain even powers of $u$ only. Nevertheless, we may use the usual form (31) of the perturbation expansion taking into account simple additional normalization in the functional-integral representation (11) for the Green functions.

The fluctuation determinant is also the same as in the massless static $\phi^{4}$ model, because it is given by the Gaussian integral with the quadratic form

$$
\frac{1}{2} \delta^{2} S=\frac{1}{2} \delta \phi\left(-\nabla^{2}+\frac{g}{2} \phi_{s t}^{2}+v_{2} m_{s t}\right) \delta \phi+\delta \phi v_{2} \phi_{s t} \delta m+\frac{1}{2} \delta m^{2}
$$

Here, the gaussian integral over $\delta m$ gives the determinant of the unit operator and produces the Gaussian integral over $\delta \phi$ with the quadratic form

$$
\frac{1}{2} \delta^{2} S=\frac{1}{2} \delta \phi\left(-\nabla^{2}+\frac{g-3 v_{2}^{2}}{2} \phi_{s t}^{2}\right) \delta \phi=\frac{1}{2} \delta \phi\left(-\nabla^{2}-\frac{\tilde{g} u_{s t}^{2}}{2} \phi_{s t}^{2}\right) \delta \phi
$$

which is exactly that of the massless $\phi^{4}$ model. A subsequent integration over $\delta u$ yields the additional factor $1 / \sqrt{1-3 \varkappa^{2}}$. 
Equation (15) for the dynamic instanton here assume the form

$$
\begin{aligned}
\frac{\partial \phi}{\partial t} & =\Gamma\left(-\nabla^{2} \phi-\frac{1}{6} \phi^{3}-i \varkappa m \phi\right) \\
\frac{\partial m}{\partial t} & =-\lambda \nabla^{2}\left(m-\frac{i}{2} \varkappa \phi^{2}\right)
\end{aligned}
$$

The zeroth-order approximation of the iterative solution can be written as

$$
\begin{aligned}
\phi_{D}^{(0)}(t, \mathbf{x}) & =\int d \mathbf{x}^{\prime} G\left(T-t, \mathbf{x}-\mathbf{x}^{\prime}\right) \phi_{s t}\left(\mathbf{x}^{\prime}\right) \\
m_{D}^{(0)}(t, \mathbf{x}) & =\frac{i \varkappa}{2} \int d \mathbf{x}^{\prime} G\left(T-t, \mathbf{x}-\mathbf{x}^{\prime}\right) \phi_{s t}^{2}\left(\mathbf{x}^{\prime}\right)
\end{aligned}
$$

where

$$
G(t, \mathbf{x})=\Theta(-t) \frac{1}{(4 \pi \Gamma|t|)^{d / 2}} \exp \left(-\frac{\mathbf{x}^{2}}{4 \Gamma|t|}\right)
$$

is the advanced diffusion kernel which determines the propagator in the treegraph solution here.

\subsection{Model D}

Model D has the same static action (23) as model C, but the kinetic coefficient is slightly different:

$$
\alpha=\left(\begin{array}{cc}
-\lambda \nabla^{2} & 0 \\
0 & -\lambda_{1} \nabla^{2}
\end{array}\right) .
$$

The matrix element $\alpha_{11}$ of this kinetic coefficient is the same as in model B.

Therefore, the analysis of the previous section 4.2 is valid here in full extent with the only replacement of the diffusion propagator $G$ for the $\phi$ field from (35) by the "hyperdiffusion" propagator $\widetilde{G}$ from relation $(22)$ in the first equation of set (34) and subsequently in the tree-graph expansion for $\phi_{d}$. Therefore, the constant $a_{D}$ is equal to $a_{c}$.

\subsection{Model F}

In model $\mathrm{F}$ there are two complex conjugate fields: $\varphi_{1}=\psi, \varphi_{2}=\psi^{*}$ and a real field $\varphi_{3}=m$. The static action is of the form:

$$
S_{F}=|\nabla \psi|^{2}+\frac{g}{6}|\psi|^{4}+\frac{m^{2}}{2}+v_{2} m|\psi|^{2}
$$


The kinetic coefficient $(\alpha+\beta)$ is determined by the matrices

$$
\begin{gathered}
\alpha=\left(\begin{array}{ccc}
0 & \lambda_{\psi} & 0 \\
\lambda_{\psi} & 0 & 0 \\
0 & 0 & -\lambda_{m} \nabla^{2}
\end{array}\right), \\
\beta=\left(\begin{array}{ccc}
0 & i v_{3} & i v_{4} \psi \\
-i v_{3} & 0 & -i v_{4} \psi^{*} \\
-i v_{4} \psi^{*} & i v_{4} \psi & 0
\end{array}\right) .
\end{gathered}
$$

The static instanton is determined by the set of equations (25), (26) and (27) of the model $\mathrm{C}$, where the filed $\phi$ is treated as a two-component one with the components $\psi^{*} / \sqrt{2}$ and $\psi / \sqrt{2}$ and $\phi^{2}=\psi^{*} \psi / 2$. Then the constant $a=a_{F}$ in relation (20) for this model was determined in section 4.2. The fluctuation determinant is also the same as in the model $\mathrm{C}$ and coincides with that of the massless static $\phi^{4}$ model.

Equation (15) for the dynamic instanton is here

$$
\begin{aligned}
\frac{\partial \psi}{\partial t} & =\left(\lambda_{\psi}+i v_{3}\right)\left(-\nabla^{2} \psi+\frac{g}{3}|\psi|^{2} \psi+v_{2} m \psi\right)-i v_{4} \psi\left(m+v_{2}|\psi|^{2}\right) \\
\frac{\partial \psi^{*}}{\partial t} & =\left(\lambda_{\psi}+i v_{3}\right)\left(-\nabla^{2} \psi^{*}+\frac{g}{3}|\psi|^{2} \psi^{*}+v_{2} m \psi^{*}\right)+i v_{4} \psi^{*}\left(m+v_{2}|\psi|^{2}\right) \\
\frac{\partial m}{\partial t} & =-\lambda_{m} \nabla^{2}\left(m+v_{2}|\psi|^{2}\right)-i v_{4} \nabla\left(\psi^{*} \nabla \psi-\psi \nabla \psi^{*}\right)
\end{aligned}
$$

with coefficients $g=-1, v_{2}=-i \varkappa, v_{4} \rightarrow-i v_{4} / u$. The iterative solution can be written here in analogy with the model $\mathrm{C}$ using the advanced propagator $G$ from (35).

\subsection{Model E}

Model $\mathrm{E}$ is $\mathrm{F}$ model with $v_{2}=v_{3}=0$. Thus, it has been analyzed in the previous section. The constant $a_{E}$ in relation (20) is equal to $a_{0}$ due to $v_{2}=0$. The static instanton for the field $m$ is $m_{s t}=0$, but a nontrivial dynamic instanton for this field may be obtained from equations (39) due to mode coupling. 


\subsection{Model $G$}

In model $\mathrm{G}$ there are two real vector fields: let $\varphi_{a}=\phi_{a}$ and $\varphi_{3+a}=m_{a}$, where $a=1,2,3$. The static action is of the form:

$$
S_{G}=\frac{1}{2}(\nabla \phi)^{2}+\frac{g}{4 !} \phi^{4}+\frac{m^{2}}{2}
$$

and the kinetic coefficient is determined by the relations

$$
\begin{gathered}
\alpha=\left(\begin{array}{cc}
\lambda_{\phi} & 0 \\
0 & -\lambda_{m} \nabla^{2}
\end{array}\right) . \\
\beta_{a b}=0, \quad \beta_{a 3+b}=v_{2} \epsilon_{a b c} \phi_{c}, \quad \beta_{3+a 3+b}=v_{2} \epsilon_{a b c} m_{c},
\end{gathered}
$$

where $\epsilon_{a b c}$ is the completely antisymmetric tensor and all the indices $a, b, c$ assume values $1,2,3$.

The static instanton solution for this model is $\phi=\phi_{s t}, m=0$. Therefore $\tilde{g}=-1$ and the constant $a_{G}$ in relation (20) coincides with the static one for the massless $\phi^{4}$ model: $a_{G}=a_{0}$. The fluctuation determinant is also the same as in the $\phi^{4}$ model.

The dynamic-instanton equation (15) for this model can be solved as described in the preceding sections.

\subsection{Model $H$}

The static action of $\mathrm{H}$ model is of the form:

$$
S_{H}=\frac{1}{2}(\nabla \phi)^{2}+\frac{g}{4 !} \phi^{4}+\frac{m^{2}}{2}+\frac{1}{2} v_{2} m \phi^{2}+\frac{c}{2} \mathbf{v}^{2},
$$

with the scalar fields $\phi$ and $m$ and the transversal vector field $\mathbf{v}$. The static instanton solution for the scalar fields turns out to be the the same as in

model $\mathrm{C}$, whereas $\mathbf{v}_{s t}=0$. Therefore, the fluctuation determinant has been also discussed in section 4.2. But in fact the critical dynamics is described by the simplified $\mathrm{H}_{0}$ model without the field $m$, whose static action is

$$
S_{H_{0}}=\frac{1}{2}(\nabla \phi)^{2}+\frac{g}{4 !} \phi^{4}+\frac{c}{2} \mathbf{v}^{2}
$$

This means that the constant $a_{H}$ here as well as the fluctuation determinant are the same as in the usual $\phi^{4}$ model: $a_{H}=a_{0}$. 
The kinetic coefficient is given by the matrices

$$
\alpha=\left(\begin{array}{cc}
-\lambda_{\phi} \nabla^{2} & 0 \\
0 & -\lambda_{v} \nabla^{2}
\end{array}\right), \quad \beta=\left(\begin{array}{cc}
0 & v_{2} \vec{\nabla} \phi \\
-v_{2} \overleftarrow{\nabla} \phi & 0
\end{array}\right)
$$

where the transverse projection operator for the vector field $\mathbf{v}$ is implied.

Equation (15) for the dynamic instanton in the $\mathrm{H}_{0}$ model assumes the form

$$
\begin{aligned}
& \frac{\partial \phi}{\partial t}=-\lambda_{\phi} \nabla^{2}\left(-\nabla^{2} \phi-\frac{1}{6} \phi^{3}\right)-i \frac{v_{2} c}{u} \mathbf{v} \nabla \phi, \\
& \frac{\partial \mathbf{v}}{\partial t}=-\lambda c \nabla^{2} \mathbf{v}+i \frac{v_{2}}{u} \phi \nabla^{3} \phi .
\end{aligned}
$$

The iterative solution may be constructed with the use of the advanced propagators (22) and (35) [the propagator (35) for the vector field $\mathbf{v}$ taken in a convolution with the transversal projection operator, however].

\section{Conclusion}

In this paper we have shown that the instanton method is applicable to largeorder asymptotic analysis for all the near-equilibrium standard models B $\mathrm{H}$ of critical dynamics in a form which is a generalization of the recently proposed dynamic-instanton approach in model A [5]. The factorial growth of the large-order coefficients of the perturbation expansion

$$
F^{[N]}=N ! C a_{M}^{N} N^{b}
$$

has been proved for any quantity $F$ calculable in the form of a perturbative series. The constants $a_{M}$ in expression (46) has been determined for all models mentioned. The exponent $b$ in relation (46), however, depends on the quantity $F$ considered. For any particular $F$ it may be readily calculated with the use of the results of the present analysis.

For instance, it may readily be proved that the exponent $b$ in the $\varepsilon$-expansion contribution to the dynamic index $z$ is determined by the large-order asymptote of the expansion of the fixed-point value $g_{*}$ and the second term of the perturbation series of the two-point correlation function. Therefore, in the $O(n)$ symmetric dynamic theory the relation

$$
b=3+\frac{n}{2}
$$

follows for the dynamic index $z$. 
The fluctuation contribution to the amplitude factor $C$ in relation (46) has been analyzed for all models $\mathrm{B}-\mathrm{H}$ as well.

The work was supported in part by the Nordic Grant for Network Cooperation with the Baltic Countries and Northwest Russia No. FIN-20/2003, and by the Academy of Finland (Grant No. 207939). M.V.K. and M.Yu.N. acknowledge the Department of Physical Sciences of the University of Helsinki for kind hospitality. The authors are grateful to A.N. Vasil'ev for fruitful discussions.

\section{Appendix}

Let us outline the calculation of the fluctuation determinant at the instanton solution along the lines proposed for the model A [5]. Since we have extracted the $u$ dependence from the dynamic action as a prefactor in Eq. (19), due to the stationarity conditions $(12)$ - (14) the contribution from the fluctuations of $u$ is independent of the field fluctuations and factorized as well. The remaining nontrivial fluctuation integral over $\delta \varphi_{a}$ and $\delta \varphi_{a}^{\prime}$ may be written as

$$
\begin{gathered}
\delta \Sigma=\left(\iint \mathcal{D} \delta \varphi \mathcal{D} \delta \varphi^{\prime} \exp \left\{\delta \varphi_{a}^{\prime} \alpha_{a b} \delta \varphi_{b}^{\prime}-\delta \varphi_{a}^{\prime}\left[\delta_{a c} \frac{\partial}{\partial t}+\left(\alpha_{a b}+\beta_{a b}^{(0)}\right)\right] \delta \varphi_{c}\right\}\right)^{-1} \\
\times \iint \mathcal{D} \delta \varphi \mathcal{D} \delta \varphi^{\prime} \operatorname{I} \exp \left\{\delta \varphi_{a}^{\prime} \alpha_{a b} \delta \varphi_{b}^{\prime}\right. \\
-\left.\delta \varphi_{a}^{\prime}\left[\delta_{a c} \frac{\partial}{\partial t}+\left(\alpha_{a b}+\beta_{a b}\right) \frac{\delta^{2} S}{\delta \varphi_{b} \delta \varphi_{c}}+\frac{\delta \beta_{a b}}{\delta \varphi_{c}} \frac{\delta S}{\delta \varphi_{b}}\right]\right|_{\varphi_{D}} \delta \varphi_{c} \\
-\frac{1}{2} \delta \varphi_{c} \varphi_{D a}^{\prime}\left[\left(\alpha_{a b}+\beta_{a b}\right) \frac{\delta^{3} S}{\delta \varphi_{b} \delta \varphi_{c} \delta \varphi_{d}}\right. \\
\left.\left.+2 \frac{\delta \beta_{a b}}{\delta \varphi_{c}} \frac{\delta^{2} S}{\delta \varphi_{b} \delta \varphi_{d}}+\frac{\delta^{2} \beta_{a b}}{\delta \varphi_{c} \delta \varphi_{d}} \frac{\delta S}{\delta \varphi_{b}}\right]\left.\right|_{\varphi_{D}} \delta \varphi_{d}\right\}
\end{gathered}
$$

where $\beta_{a b}^{(0)}$ is the field-independent part of the kinetic coefficient $\beta$ (we remind that the kinetic coefficient $\alpha$ is assumed to be field-independent). The change of variables

$$
\delta \varphi_{a}^{\prime}=\delta \psi_{a}^{\prime}+\left(\alpha^{-1}\right)_{a b}\left(\frac{\partial \delta \varphi_{b}}{\partial t}+\beta_{b c} \frac{\delta^{2} S}{\delta \varphi_{c} \delta \varphi_{d}} \delta \varphi_{d}+\frac{\delta \beta_{b c}}{\delta \varphi_{d}} \frac{\delta S}{\delta \varphi_{c}} \delta \varphi_{d}\right)
$$

together with the antisymmetry of the kinetic coefficient $\beta$, the instanton equation (15) and the second equality in relation (16) then allows to express the quadratic form of the exponential of the numerator of fluctuation integral 
(47) as

$$
\begin{aligned}
-\frac{1}{2} \delta_{\left\{\varphi, \varphi^{\prime}\right\}}^{2} \bar{S} & =\delta \psi_{a}^{\prime} \alpha_{a b} \delta \psi_{b}^{\prime} \\
-\delta \psi_{a}^{\prime} & \left\{-\frac{\partial \delta \varphi_{a}}{\partial t}+\left.\left[\left(\alpha_{a b}-\beta_{a b}\right) \frac{\delta^{2} S}{\delta \varphi_{b} \delta \varphi_{c}}-\frac{\delta \beta_{a b}}{\delta \varphi_{c}} \frac{\delta S}{\delta \varphi_{b}}\right]\right|_{\varphi_{D}} ^{\delta \varphi_{c}}\right\} \\
& -\left.\frac{1}{2} \delta \varphi_{s t a} \frac{\delta^{2} S}{\delta \varphi_{a} \delta \varphi_{b}}\right|_{\varphi_{s t}} \delta \varphi_{s t b}+\left.\frac{1}{2} \delta \varphi_{0} \frac{\delta^{2} S}{\delta \varphi_{a} \delta \varphi_{b}}\right|_{\varphi_{0}} \delta \varphi_{0 b}
\end{aligned}
$$

where $\delta \varphi_{s t a}=\left.\delta \varphi_{a}\right|_{t=T}$ and $\delta \varphi_{0 a}=\left.\delta \varphi_{a}\right|_{t=t_{0}}$. To calculate the fluctuation integral perturbatively, we write - using the change of variables (48) - the normalization factor in terms of the perturbation expansion implied in (49):

$$
\begin{aligned}
-\frac{1}{2} \delta_{\left\{\varphi, \varphi^{\prime}\right\}}^{2} \bar{S}_{0}=\delta \psi_{a}^{\prime} \alpha_{a b} \delta \psi_{b}^{\prime}-\delta \psi_{a}^{\prime} & {\left[-\delta_{a c} \frac{\partial}{\partial t}+\left(\alpha_{a b}-\beta_{a b}^{(0)}\right) K_{b c}\right] \delta \varphi_{c} } \\
& -\frac{1}{2} \delta \varphi_{s t a} K_{a b} \delta \varphi_{s t b}+\frac{1}{2} \delta \varphi_{0 a} K_{a b} \delta \varphi_{0 b}
\end{aligned}
$$

with the same free-field part for the perturbative calculation as in (49). This representation gives rise to the propagators $\Delta_{12}=\Delta_{21}^{\top}$ and the correlation function $\Delta_{11}$ determined by the operator $M_{0}$ [defined by equation (7)], its transpose $M_{0}^{\top}$ and the correlation function of the Langevin source (2) as

$$
\begin{aligned}
& \left(\Delta_{12}\right)_{a b}\left(t, \mathbf{x} ; t^{\prime}, \mathbf{x}^{\prime}\right)=\left(M_{0}^{-1}\right)_{a b}\left(t, \mathbf{x} ; t^{\prime}, \mathbf{x}^{\prime}\right), \\
& \left(\Delta_{11}\right)_{a b}\left(t, \mathbf{x} ; t^{\prime}, \mathbf{x}^{\prime}\right)=2\left(\Delta_{12} \alpha \Delta_{21}\right)_{a b}\left(t, \mathbf{x} ; t^{\prime}, \mathbf{x}^{\prime}\right),
\end{aligned}
$$

where $\Delta_{12}$ is the retarded Green's function of the operator $M_{0}$ (and, correspondingly, $\Delta_{21}$ the advanced Green's function).

Inspection of the Fourier transform of the propagator $\left(\Delta_{12}\right)_{a b}\left(t, \mathbf{x} ; t^{\prime}, \mathbf{x}^{\prime}\right)$ shows that that in the time-space representation it is rapidly decaying at $t-t^{\prime} \rightarrow \infty$, which, together with the initial condition $\lim _{t-t^{\prime} \rightarrow 0_{+}}\left(\Delta_{12}\right)_{a b}\left(t, \mathbf{x} ; t^{\prime}, \mathbf{x}^{\prime}\right)=\delta_{a b} \delta(\mathbf{x}-$ $\mathbf{x}^{\prime}$ ), is basically all what is needed for an analysis similar to that for model A $[5]$.

Let us introduce for the numerator of relation (47) the following decomposition of the integration variable

$$
\delta \varphi_{a}=\overline{\delta \varphi}_{a}+\delta \varphi_{a}^{T}
$$

where $\overline{\delta \varphi}$ consists of the standard Gaussian integration variable constructed with the aid of the advanced Green's function $\left(\Delta_{21}\right)_{a b}$ and the solution $h$ of the homogenous equation $\left(M_{0}^{\top}\right)_{a b} h_{b}(t, \mathbf{x})=0$ such that $\overline{\delta \varphi}_{a}(T, \mathbf{x})=0$, i.e.

$$
\overline{\delta \varphi}_{a}=\left(\Delta_{11}\right)_{a b} \eta_{b}+\left(\Delta_{21}\right)_{a b} \eta_{b}^{\prime}+h_{a}
$$


where $\eta_{a}$ and $\eta_{a}^{\prime}$ are fields vanishing at the boundaries (i.e. at $|\mathbf{x}| \rightarrow \infty, t=T$ and $\left.t=t_{0}\right)$. This choice is accompanied by the prescription

$$
\delta \psi_{a}^{\prime}=\left(\Delta_{12}\right)_{a b} \eta_{b}
$$

for the auxiliary field.

The second term $\delta \varphi^{T}$ in the decomposition (53) is chosen as the solution of the homogeneous equation

$$
\left.\left[-\delta_{a c} \frac{\partial}{\partial t}+\left(\alpha_{a b}-\beta_{a b}\right) \frac{\delta^{2} S}{\delta \varphi_{b} \delta \varphi_{c}}-\frac{\delta \beta_{a b}}{\delta \varphi_{c}} \frac{\delta S}{\delta \varphi_{b}}\right]\right|_{\varphi_{D}} \delta \varphi_{c}^{T}(t, \mathbf{x})=0
$$

with the final value $\delta \varphi_{a}^{T}(T, \mathbf{x})=\delta \varphi_{\text {sta }}(\mathbf{x})$ to conform to the boundary condition. The solution of equation (56) can be constructed perturbatively using the tree-graph expansion of $\varphi_{D}$.

The integration space for the normalization factor in the denominator of relation (47) is constructed with the aid of a decomposition similar to (53) with the same $\overline{\delta \varphi}$ and $\delta \psi_{a}^{\prime}$. In this case, however, the second term $\delta \varphi^{T}$ in the decomposition may be expressed explicitly as the spatial convolution of the advanced Green's function $\left(\Delta_{21}\right)_{a b}\left(t, \mathbf{x} ; T, \mathbf{x}^{\prime}\right)$ (note the fixed time argument) and the function $\delta \varphi_{\text {sta }}(\mathbf{x})$ and thus is the solution of the free-field counterpart of equation (56):

$$
\left[-\delta_{a c} \frac{\partial}{\partial t}+\left(\alpha_{a b}-\beta_{a b}^{(0)}\right) K_{b c}\right] \delta \varphi_{c}^{T}(t, \mathbf{x})=0 .
$$

With the use of the asymptotic properties of the integration variables constructed in this manner it may be shown that in the limit $T \rightarrow \infty$ the final (static) fluctuations factorize completely (as in the case of model A):

$$
\begin{aligned}
& \delta \Sigma=\left[\int \mathcal{D} \delta \varphi_{s t} \exp \left(-\frac{1}{2} \delta \varphi_{s t a} K_{a b} \delta \varphi_{s t b}\right)\right]^{-1} \\
& \times \int \mathcal{D} \delta \varphi_{s t} \mathrm{I} \exp \left(-\left.\frac{1}{2} \delta \varphi_{s t a} \frac{\delta^{2} S}{\delta \varphi_{a} \delta \varphi_{b}}\right|_{\varphi_{s t}} \delta \varphi_{s t b}\right)\left(\int \int \mathcal { D } \overline { \delta \varphi } \mathcal { D } \delta \psi ^ { \prime } \operatorname { e x p } \left\{\delta \psi_{a}^{\prime} \alpha_{a b} \delta \psi_{b}^{\prime}\right.\right. \\
& \left.\left.-\delta \psi_{a}^{\prime}\left[-\frac{\partial \overline{\delta \varphi}_{a}}{\partial t}+\left(\alpha_{a b}-\beta_{a b}^{(0)}\right) K_{b c} \overline{\delta \varphi_{c}}\right]+\frac{1}{2} \overline{\delta \varphi}_{0 a} K_{a b} \overline{\delta \varphi_{0 b}}\right\}\right)^{-1} \\
& \times \iint \mathcal{D} \overline{\delta \varphi} \mathcal{D} \delta \psi^{\prime} \exp \left\{\delta \psi_{a}^{\prime} \alpha_{a b} \delta \psi_{b}^{\prime}-\delta \psi_{a}^{\prime}\left[-\frac{\partial \overline{\delta \varphi}_{a}}{\partial t}+\left(\alpha_{a b}-\beta_{a b}\right) \frac{\delta^{2} S}{\delta \varphi_{b} \delta \varphi_{c}}\right.\right. \\
& \left.\left.-\frac{\delta \beta_{a b}}{\delta \varphi_{c}} \frac{\delta S}{\delta \varphi_{b}}\right]\left.\right|_{\varphi_{D}}{\overline{\delta \varphi_{c}}}_{1}+\frac{1}{2}{\overline{\delta \varphi_{0}}}_{0} \frac{\delta^{2} S}{\delta \varphi_{a} \delta \varphi_{b}}||_{\varphi_{0}}{\overline{\delta \varphi_{0 b}}}_{\}}\right\}
\end{aligned}
$$


where ${\overline{\delta \varphi_{0}}}_{a}=\left.\overline{\delta \varphi}_{a}\right|_{t=t_{0}}$. On the right-hand-side of (57) the first two factors yield the static fluctuation determinant including the degeneracy-lifting unit decomposition. A non-trivial dependence on the initial time instant $t_{0}$ remains in (57) through the non-zero initial value of the fluctuation field ${\overline{\delta \varphi_{0}}}_{0}$. When the translation invariance with respect to time is restored by passing to the limit $t_{0} \rightarrow-\infty$, however, the initial value of the fluctuation field vanishes: $\bar{\delta}_{0} \rightarrow 0$ due to the structure of the integration field (53) and the attenuation property of the advanced Green function. In a manner similar to that used for model A [5] it may then be shown perturbatively that in this limit the dynamic contribution to the fluctuation integral (57) tends to unity and thus we arrive at the fluctuation integral

$$
\lim _{\substack{T \rightarrow \infty \\ t_{0} \rightarrow-\infty}} \delta \Sigma=\frac{\int \mathcal{D} \delta \varphi_{s t} \mathrm{I} \exp \left(-\left.\frac{1}{2} \delta \varphi_{s t a} \frac{\delta^{2} S}{\delta \varphi_{a} \delta \varphi_{b}}\right|_{\varphi_{s t}} \delta \varphi_{s t b}\right)}{\int \mathcal{D} \delta \varphi_{s t} \exp \left[-\frac{1}{2} \delta \varphi_{s t a} K_{a b} \delta \varphi_{s t b}\right]}
$$

completely determined by the static theory.

\section{References}

[1] L.N. Lipatov, Zh. Eksp. Teor. Fiz. 72 (1977) 411.

[2] E.Brezin, J.C. Le Guillou and J. Zinn-Justin, Phys. Rev. D 15 (1977) 1544.

[3] M.V. Komarova and M.Yu. Nalimov, Theor. Math. Phys. 126 (2001) 393.

[4] P.C. Martin, E.D. Siggia and H.A. Rose, Phys. Rev. A 8 (1973) 423.

[5] J. Honkonen, M.V. Komarova and M.Yu. Nalimov, hep-th/0406168.

[6] P.C. Hohenberg and B.I. Halperin, Rev. Mod. Phys. 49 (1977) 435.

[7] A.N. Vasil'ev, The Field Theoretic Renormalization Group in Critical Behavior Theory and Stochastic Dynamics (Chapman \& Hall/CRC, Boca Raton, 2004).

[8] C. De Dominicis, J. Phys. (Paris) 37, Suppl C1 (1976) 247;

H.K. Janssen, Z. Phys. B 23 (1976) 377.

[9] A.N. Vasiliev, Functional Methods in Quantum Field Theory and Statistical Physics (Gordon and Breach, Amsterdam, 1998).

[10] J. Zinn-Justin, Quantum Field Theory and Critical Phenomena (Clarendon Press, Oxford, 1996).

[11] L.M. Suslov, JETP 84 (1997) 120.

[12] M.V. Komarova and M.Yu. Nalimov, Theor. Math. Phys. 129 (2001) 387. 